\title{
DEUDA EXTERNA SOBERANA:DRAMA SOCIAL LATINOAMERICANO EN LA DÉCADA DE 1980 Y TRAGEDIA DE LA EUROPA POSTMODERNA EN LA DÉCADA DE 2000
}

\author{
ROBERTO POSSO ORDÓÑEZ \\ Catedrático universitario y Consultor Económico
}

\section{RESUMEN}

Permítanme explicar el por qué del título de esta ponencia. Si bien el mismo hace alusión a dos géneros teatrales originarios de la Grecia Antigua antes que a un problema vinculado con la deuda externa soberana de los países de nuestro planeta, se ha utilizado esta figura para asemejar al drama a la crisis de la deuda externa de América Latina de finales del siglo XX y como tragedia a la crisis de la deuda iniciada en Europa a comienzos del siglo XXI. Desde la perspectiva de los géneros teatrales, un "drama" es la representación de una acción de la vida cotidiana en la que los personajes que intervienen en él, utilizan el diálogo para tratar asuntos serios y profundos. La "tragedia" es una obra cuyo contenido causa terror y hay un desenlace funesto. En este género teatral, como en la época de Esquilo o Sófocles, intervienen personajes ilustres o heroicos, mientas que para Sófocles sus personajes son personas problemáticas e inseguras, muy alejadas del modelo clásico. Para completar el panorama sombrío de la actual tragedia, nosotros -los seres humanos- somos parte de los protagonistas y quienes sufriremos las consecuencias de la crisis de la deuda griega y de la recesión mundial, caso de llegar a suscitarse.

Palabras Clave: Deuda externa, drama, tragedia.

\begin{abstract}
Let me explain the reason for the title of this paper. Although it refers to two theatrical genres originating in ancient Greece before a problem linked to the sovereign debt of the countries of our planet, this figure has been used to resemble the drama to the crisis of the external debt of Latin America at the end of the 20th century and as a tragedy to the debt crisis started in Europe at the beginning of the 21st century. From the perspective of theatrical genres, a "drama" is the representation of an action of everyday life in which the characters involved in it, use dialogue to address serious and profound issues. "Tragedy" is a work whose content causes terror and a fatal outcome. In this theatrical genre, as in the time of Aeschylus or Sophocles, illustrious or heroic characters involved, while that for Sophocles, its characters are people problems and unsafe, too far away from the classical model. To complete the bleak panorama of the current tragedy, we - humans - are part of the protagonists and who will suffer the consequences of the crisis of the Greek debt and the global recession, case to arise.
\end{abstract}

Key words: External debt, drama and tragedy. 


\section{COMIENZA LA FUNCIÓN}

¡Arriba el telón! El documento aborda tres aspectos referentes a la deuda externa. La primera parte tiene una connotación histórica y describe la crisis de la deuda de América Latina de finales del siglo pasado, con un enfoque "ortodoxo" acerca del rol determinante, en la crisis, de los desequilibrios internacionales generados por los países industrializados. Se mencionan a esos desequilibrios como un efecto del régimen dominante de acumulación de capital a nivel mundial, en cuya estructuración juegan un rol esencial los estados centrales -o economías desarrolladas- y las exigencias del capital transnacional. Los países desarrollados fueron la principal fuente de capital financiero en busca de posibilidades de inversión. Después del aumento del precio internacional del petróleo, algunos países miembros de la OPEP también colocaron sus excedentes de capital en los mercados financieros. No hay que perder de vista que hasta fines de la década de los 1970, las tasas de interés internacionales fueron bajas y hasta podría decirse que en términos reales fueron negativas.

En la segunda parte se examina, partiendo de esta misma óptica, los elementos determinantes previos al estallido de la actual crisis de la deuda europea, poniendo énfasis en los mecanismos financieros que sostuvieron y estimularon el endeudamiento en Europa. Igualmente se examina, con algún detalle, el comportamiento económico de los gobiernos helénicos, cuya "actuación teatral" generó y está desencadenando la crisis motivo de este análisis. Grecia ha demostrado como el sobreendeudamiento, más allá de los niveles técnicos aconsejados, se constituye en una bola de nieve que atenta no solo contra su propio desarrollo económico sino el de todos los países de Europa y quizá del mundo entero.

No sólo el sobreendeudamiento que financió el desarrollo fue el inicio de la crisis. La corrupción de las autoridades griegas; la evasión de impuestos por parte de las grandes corporaciones; la moratoria, desfases y trampas a la banca, con consentimiento político, fueron factores que también coadyuvaron a este desenlace. Los juegos olímpicos de 2004 hicieron que aumente el gasto del gobierno y el "maquillaje" hecho a las cuentas gubernamentales, se añaden como causas de esta crisis.

En la tercera sección, a modo de conclusiones, se resaltan hechos ocurridos tanto en la crisis de la deuda de latinoamericana como en la europea y se hacen comentarios de algunos de los principales acontecimientos ocurridos en los últimos años del siglo $\mathrm{XX}$, así como en el primer decenio del siglo XXI, con el fin de contribuir para aclarar algunos eventos que la historia de la crisis de deuda los recoge en sus páginas y los hechos que deberá registrarlos en la semblanza que comienza a escribirse para el capítulo europeo.

\section{LA CRISIS DEL ENDEUDAMIENTO EXTERNO LATINOAMERICANO DE FINES DEL SIGLO XX}

Joachim Becker, de manera muy singular sintetiza lo que sucedía en América Latina durante la década de los setenta con los créditos externos, al manifestar que "El endeudamiento 
parecía una 'oferta' barata”'. En efecto, la acumulación de capital en los países desarrollados provocó un exceso de liquidez internacional. El excesivo endeudamiento de los países de América del Sur, iniciado en la década de los setenta, tiene las siguientes características: i) se origina por las facilidades de financiamiento concedidas por los países capitalistas desarrollados; ii) por la afluencia de capital proporcionado por parte de las empresas multinacionales a los países latinoamericanos, iii) bajas tasas internacionales de interés (más bajas que las registradas para los préstamos de los años cincuenta $^{2}$ ) y, iv) la no condicionalidad impuesta a créditos externos por organismos internacionales -en los cuales tienen mayor influencia Estados Unidos, Japón y Europa-. Después de los sucesivos aumentos del precio internacional del petróleo, ocurridos en la década de los setenta, algunos de los países miembros de la OPEP también colocaron sus excedentes en los mercados financieros.

El endeudamiento externo, generalmente ligado al comercio, condujo a un extraordinario crecimiento económico y a "cierta" estabilidad económica. Efectivamente, según el Banco Interamericano de Desarrollo (BID), la tasa de crecimiento real promedio anual del PIB de los países de América Latina, en el período comprendido entre $1970 \mathrm{y}$ 1979, llegó al 5,9 por ciento ${ }^{3}$. En con-

1. Becher, Joachim (2007). El endeudamiento externo de América Latina: un resumen histórico, en: "El golpe del capital. Las crisis financieras en el Cono Sur y sus salidas". Montevideo: Editorial Coscoroba, p. 17.

2. Ibíd., p. 16.

3. Banco Interamericano de Desarrollo (Octubre de 1992). Progreso Económico y Social en América Latina Informe 1992. Washington D.C., p. 2. secuencia, el desarrollo económico de los países latinoamericanos continuó dependiendo de las importaciones de bienes de capital, habiendo una marcada tendencia a registrar déficit en la cuenta corriente de la Balanza de Pagos. No obstante lo anterior, el modelo de sustitución de importaciones adoptado por los países de la Región se agotó y quedó incompleto “...porque ni el capital nacional ni el capital internacional estaban interesados en invertir en este sector"4.

Adicionalmente, se puede afirmar que no todos los recursos financieros provenientes del exterior se utilizaron de manera adecuada o con la finalidad de mejorar la calidad de vida de la población pues una parte importante de los mismos se destinaron al pago de intereses de la deuda externa y a la compra de armamento.

Por otra parte, debemos recordar que a comienzos de los años setenta se consolida el mercado de los eurodólares y por coincidencia, en esa misma época, comienza a sentirse la crisis de la hegemonía de los Estados Unidos. Esta decadencia no solo fue en lo económico, sino también aparece en los campos político y militar. Estas circunstancias incidieron en el debilitamiento del dólar como moneda que sostenía el sistema de tipo de cambio fijo creado con el convenio de Bretton Woods y que estuvo vigente desde 1946.

A finales de la década de los setenta, Latinoamérica siente, por un lado la presión que ejercen los acreedores que exigían el pago del servicio de la deuda y por otro, la fuga de capitales

4. Becker, Op. cit., p. 16. 
de la Región, estimulada por el alza de las tasas de interés registrada en los Estados Unidos.

EL FMI y el Banco Mundial estiman que la deuda externa latinoamericana, en la década de los setenta creció alrededor de 130.000 millones de dólares, pues según esas fuentes, a finales de 1970 el saldo del endeudamiento era aproximadamente 29.000 millones mientras que a fines de 1979 alcanzaron los 159.000 millones de dólares ${ }^{5}$. Es decir que el incremento anual promedio fue de cerca de 15.000 millones, esto es un monto equivalente a casi el 50 por ciento del saldo deudor registrado en diciembre de 1970 .

En la década de los años 80, los efectos negativos del sobreendeudamiento ya se hicieron evidentes, pues se agudizaron los problemas económicos y sus resultados se reflejan en el comportamiento de las variables macroeconómicas tales como tasas de desempleo, salarios reales e incremento de precios. (Ver anexo "Variación anual en el índice de precios al consumidor, 1971-1991”),

Según los registros del BID respecto de los saldos de la deuda externa total desembolsada, se aprecia que entre 1982 y 1989, éstos se incrementaron en 89.756 millones de dólares, lo que significa que en ese período la deuda externa de América Latina creció a una tasa anual promedio de alrededor del 27 por ciento, no obstante que la crisis de la deuda se agudizó a mediados de 1982 (Ver anexo "Saldo de la deuda externa total desembolsada") y que supuestamente los procesos de

5. Hernández, Gladys. Evolución de la deuda externa de América Latina y el Caribe, en: www.oid-ido.org, 16-11-2005. renegociación de la misma impidieron el libre flujo de capitales.

En agosto de 1982, México anuncia una moratoria en el pago de su deuda externa, desatando, así, la crisis de la deuda en América Latina ${ }^{6}$ y afectando, cada vez más, al desarrollo económico de la Región. Los procesos de refinanciamiento de la deuda externa, obligaron a los países latinoamericanos a someterse a severos ajustes económicos oficiosamente impuestos por las instituciones financieras internacionales. En definitiva, el sobreendeudamiento y la imposibilidad de pago significaron que la población de los respectivos países sea la que pague las consecuencias de ese endeudamiento. Esta es la razón por la cual la Comisión Económica para América Latina (CEPAL), calificó a este decenio como la "década perdida".

La crisis de la deuda de los países del tercer mundo puso a la banca privada de los países acreedores en una situación precaria, siendo posiblemente los bancos de los Estados Unidos los que tuvieron los mayores problemas. En el artículo escrito por Joachim Becker y que aparece en el libro "El golpe del capital. Las crisis financieras en el Cono Sur y sus salidas" el autor hace una cita de Ffrench-Davis, en la cual se demuestra el riesgo de la banca privada internacional: "En 1982 el coeficiente préstamo/capital de nueve de los bancos más grandes de los Estados Unidos era el 180 por 100; de esta cifra, los préstamos vigentes en México representaban el 50 por 100, en Brasil de 46 por 100, en Venezuela, el 26 por 100, en Argentina, el 21 por 100, y en Chile, el 12 por 100. Los bancos bri-

6. Becker. Op. cit., p. 22. 
tánicos prestamistas de la región seguían un patrón similar"7. Los bancos alemanes tuvieron una mejor suerte que sus similares norteamericanos.

La renegociación de la deuda externa estuvo sujeta a un acuerdo previo de cada país con el FMI. La mencionada institución impuso políticas de ajuste estructural encaminadas a obtener superávit en la balanza comercial con el fin de obtener recursos financieros que permitieran a los países deudores pagar por lo menos los intereses de su deuda externa. Para lograr ese objetivo, algunos países utilizaron la devaluación de su moneda local, con lo cual se disminuían las importaciones y se benefició al sector exportador. En la mayoría de los casos, las políticas de ajuste a las que debieron someterse los países deudores tuvieron relación con aspectos fiscales, monetarios y salariales y estuvieron, también, encaminadas a restringir la demanda interna e igualmente generar excedentes que les permitiera pagar la deuda externa. Es interesante recalcar que las políticas de ajuste tuvieron una excepción para su aplicación y ésta se refiere a la "nacionalización" de la deuda privada, es decir que el gobierno asumía la obligación privada y garantizaba su pago. La "nacionalización" de la deuda privada fue una condición impuesta por los acreedores dentro del proceso de renegociación de la deuda. Como estas políticas de ajuste contrajeron las tasas de crecimiento de la actividad económica de los países latinoamericanos, podemos afirmar, entonces, que la crisis de la deuda aceleró la marcha hacia la adopción de modelos neoliberales.

7. Ibídem.
Refiriéndonos cronológicamente a los cambios en los modelos de renegociación de la deuda externa, podemos señalar que a inicios de 1985 y en marco del "Plan Baker", se combinó la reestructuración de la deuda con la concesión de nuevos préstamos tanto por parte del sector privado de los países acreedores así como de los organismos internacionales. En 1987 se permitió “...una reducción de la deuda en forma de readquisición de la deuda con respecto del valor facial, de 'bonos de salida' con tipos de interés bajos y de canjes de la deuda por otros motivos"'. En 1989 se innovó el plan "Baker", para permitir a los países deudores la recompra de sus propios pagarés, a precios de mercado. El aflojamiento de la condicionalidad en los planes de reestructuración de la deuda externa influyó en el ánimo de los inversionistas, quienes consideraron que podrían volver a confiar en los países de la Región.

\section{LA TRAGEDIA DE LA CRISIS DE LA DEUDA EXTERNA EN LA EUROPA POSTMODERNA}

Grecia se incorpora a la Comunidad Europea en 1981, en 1986 se adhieren España y Portugal, mientras Irlanda era integrante de la misma desde 1973, luego de una consulta interna que se realizó en el mencionado país. A mediados del año 2000 la Comisión Europea ${ }^{9}$ anunció que Grecia podría ingresar a la zona del euro en enero de 2001.

8. Becker, Op. cit., p. 23.

9. La Comisión Europea es el órgano ejecutivo de la UE y representa los intereses de Europa, no los de país alguno en concreto. Cada 5 años se nombra a un comisario por cada país miembro. 
Estudiosos del problema de la deuda externa europea estiman que la misma se ha generado como consecuencia de los múltiples errores que se cometieron en la creación de la unión monetaria. Consideran que como paso previo a la formación de aquella, los países debieron haberse constituido como un área económica homogénea y haber armonizado sus políticas fiscales, que hubiese permitido alcanzar austeridad en el gasto público con presupuestos equilibrados y controlado endeudamiento soberano. Manifiestan, además que desde la perspectiva de "solidez" económica, en la UE existen dos grupos de países claramente diferenciados (Alemania y Francia, por un lado y los países PIGS, por otro) y critican la política monetaria expansiva seguida por el Banco Central Europeo, especialmente desde el año $2001^{10}$. Estos economistas consideran que "...la zona euro desde su creación no era una zona monetaria óptima, defienden que el Pacto de estabilidad y crecimiento no se aplicó con la suficiente firmeza (Alemania desde el principio incumplió los criterios para mantenerse en la zona euro). Además Grecia mintió para entrar en la eurozona con lo que no estaba preparada en realidad para su ingreso en la unión monetaria. Cuando Yorgos Papandreu ganó las elecciones develó un déficit del 12,7 \% del PIB en detrimento del 3\% anunciado por el anterior gobierno, además anunció una Deuda pública del 120\% del PIB"11. Los porcentajes señalados por Papandreu superan en forma alarmante los

10. Fernández, Ángel (15 de septiembre de 2011). Instituto Juan de Mariana, www.juandemariana.or/ comentario/5225/crisis/deuda/eurozona/.

11. http://es.wikipedia.org/wiki/Crisis_de_la_deuda_soberana_europea, p. 11. límites del 3\% y 60\% que fija el Pacto de estabilidad y crecimiento para los miembros de la UE.

A estos antecedentes se debe añadir que la tragedia de la deuda soberana europea, también llamada crisis del euro o crisis de la zona euro, comenzó a escribirse a comienzos de la primera década de este siglo debido a que el gobierno griego se endeudó en forma desmedida y el gasto público se disparó sobre todo por la organización de los juegos olímpicos del año 2004. Pero en el año 2002, funcionarios griegos y representantes de Goldman Sachs ${ }^{12}$ llegaron a un acuerdo beneficioso para ambas partes, pero que agrava tremendamente la crisis: la deuda soberana griega en dólares y yenes era canjeada por deuda en euros, que luego de un determinado período volvía a ser canjeada por la divisa original. Así, "el monto de la deuda griega cambiaba hasta convertirse en un espejo ideal que permitía un mayor endeudamiento del gobierno a tasas de interés relativamente bajas"13. Esta imagen fiscal comenzó a tambalear con la crisis financiera y la cercanía del vencimiento de los bonos emitidos por el Estado griego, el cual debía honrar sus deudas en su moneda original.

Todo lo señalado anteriormente provocó una repentina pérdida de confianza en la zona euro y podría aún

12. Es el grupo de inversión más grande del mundo. Fue fundado en 1869 y tiene su sede principal en el bajo Manhattan, en New York. Entre sus antiguos funcionarios están tres ex secretarios del Tesoro de los Estados Unidos, que lo fueron bajo las presidencias de Clinton y George W. Bush y Romano Prodi ex Primer Ministro italiano y ex Presidente de la Comisión Europea.

13. Justo, Marcelo, BBC Mundo,www.bbc.co.uk/mundo/ economía/2010/03/100325. 
amenazar la estabilidad financiera de toda la Unión Europea.

Quizá fue por esto que inicialmente, algunos países de la eurozona estuvieron reacios a aceptar un acercamiento con el FMI porque consideraban que una intervención de ese organismo internacional sería crear una relación de dependencia que podría dañar la credibilidad del euro ${ }^{14}$.

En marzo del 2010 se inició, entre las autoridades de la UE y del FMI, un debate para acordar un paquete de medidas destinadas a rescatar la economía griega. Las discusiones estuvieron paralizadas por algunas semanas debido al desacuerdo existente entre Alemania -economía líder de la zonay otros países miembros. El argumento de Alemania se basa en que no existe una cláusula en el tratado inicial de la UE que permita a sus países rescatar financieramente a los otros. No obstante lo anterior, los ministros financieros de la UE negociaron un plan de rescate por 750.000 millones de euros. Esto incluiría un fondo de estabilización de 500.000 millones de euros, que a su vez incluiría una recaudación de 60.000 millones de euros procedentes de bonos de la UE. Al plan de rescate la UE aporta con 500.000 millones y el FMI con 250.000 millones $^{15}$. El paquete será administrado conjuntamente por los estados miembros, la Comisión Europea y el FMI. Además, los países "mayores" se comprometieron a adoptar sus propias políticas fiscales de

\footnotetext{
14. Wikipedia. Op. cit., p. 3.

15. Acuerdan "mecanismo excepcional" para defender la eurozona. www.espanol.rfi.fr/europa/20110508,08-05-2010
}

ajuste, iniciándose así una nueva era de austeridad ${ }^{16}$.

Con cargo a esos recursos, a partir de mayo de 2010, el país helénico comenzó a recibir recursos con cargo al primer rescate de 110.000 millones de euros y en julio de 2011 el Eurogrupo aprobó un segundo rescate por 109.000 millones $^{17}$.

A diferencia de lo que sucedió en el drama que vivió América Latina, en el cual si se conocieron las cifras de endeudamiento por cada país, en la tragedia europea no se conoce con exactitud el monto de la deuda externa. Así, por ejemplo, Marcelo Justo de BBC Mundo ${ }^{18}$, el 25 de marzo de 2010 , afirma: "Hoy la deuda griega, de más 400.000 millones de euros, representa el 115\% de su PIB y su déficit fiscal, el 12,7\%”. Mientras Diario El Mercurio, de la ciudad de Cuenca, Ecuador, cita que en septiembre de 2011 la deuda helénica "supera los 350.000 millones de euros (más del 150\% de su PIB)". Muchas de las fuentes de información prefieren, entonces referirse al problema de la deuda en términos relativos y no en términos absolutos. Para tener una idea de la magnitud de la tragedia europea, citemos lo que dice el diario español Cinco Días: “...la última información disponible que ofrece el BIS referida al primer trimestre de 2011 sitúa a la banca francesa y a la alemana en los primeros puestos del ranking de exposición, concentrando en sus balances el $37 \%$ y $25 \%$, respectivamente, de la deuda externa griega (pública

16. Ibíd., p. 1.

17. www.espanol.rfi.rf/europa/20100502 "El eurogrupo da luz verde al plan de rescate.

18. BBC Mundo-Economía. ¿Quien es responsable de la crisis en Grecia? www.bbc.co.uk/mundo/economia 
y privada) en poder de los bancos...., estando en la banca europea el 93\% del total de la deuda externa griega en poder de los bancos. Afortunadamente, en esta primera rueda de impacto la exposición de la banca española es marginal (1.265 millones de dólares)". Luego continúa: “...la banca es el sector más castigado ya que es el principal tenedor de deuda externa. Si nos centramos en la banca europea, los datos del BIS muestran que en los balances de los sectores bancarios europeos hay 465.000 millones de dólares en deuda externa irlandesa, $204.600 \mathrm{mi}-$ llones en deuda portuguesa, 809.000 millones de deuda italiana y 634.000 millones de deuda española. Por lo tanto, si la banca europea tuviera que asumir las pérdidas derivadas de una merma de valor de esa deuda externa, los efectos sobre sus cuentas de resultados (vía provisiones) y sobre sus niveles de solvencia (consumo de capital) serían devastadores" ${ }^{19}$.

El reto que se plantea ahora para los países endeudados de la zona euro es el de reducir su déficit presupuestario, mientas adoptan dolorosas reformas estructurales tales como reducir la inversión pública, rebajar los salarios, aumentar impuestos, privatizar empresas públicas, reducir el tamaño de la burocracia, etc. Es decir que los pobres y los jóvenes del viejo continente son los que más sufrirán los impactos de la tragedia de la deuda. Van a pagar "justos por pecadores" y la tragedia recién comienza. Por ahora, iAbajo el telón!

19. Maudos, Joaquín. www.cincodias.com/articulo/ ipinion/tragedia- griega-banca-europea
¿ES LA HISTORIA UN INCESANTE VOLVER A EMPEZAR O RECIÉN SE ESTÁ ESCRIBIENDO LA HISTORIA DE LA DEUDA AUTÓNOMA EUROPEA?

* Si cabía alguna duda de los efectos de la globalización y la interdependencia de la economía mundial, el colapso de la economía griega ya no deja ninguna sospecha. La paciencia europea pasa factura a los países más sólidos, pues en medio de la crisis griega se hicieron evidentes las falencias de las otras economías frágiles. Ni Portugal ni Irlanda se ajustaron a los modelos de austeridad y por cierto el futuro de Italia y España no son impredecibles.

* Goldman Sachs podría caber perfectamente dentro del molde euripidiano ya que ha logrado crear propuestas financieras para amasar fortunas en medio de las ruinas.

* En América Latina las negociaciones se realizaron en forma bilateral, bajo patronazgo de los Estados Unidos (recordemos que el FMI, es una ventanilla adicional del gobierno de la unión americana). En Europa las negociones se realizan entre técnicos de la UE, FMI y Banco Central Europeo (BCE) -llamada la "troika"- por un lado y por otro los representantes del respectivo país, con preeminencia de los representantes de la UE.

* En Latinoamérica se "estatizaron" las deudas privadas. El sector privado aprovechó esta circunstancia para utilizar los recursos que les quedaron libres para comprar las empresas públicas progresivamente privatizadas. Este mecanismo lo utilizaron a partir del segundo quinquenio de la década de los ochen- 
ta $^{20}$. En Europa no se conoce, por el momento, que el sector privado vaya a utilizar esta metodología.

* Los países de la Región utilizaron como mecanismo para "obtener recursos" el no pago de sus obligaciones. Esos fondos los utilizaron para financiar proyectos de inversión que estaban inconclusos o para iniciar nuevos proyectos. En Europa todavía no se presenta una coyuntura similar.

* En América latina se utilizó el mecanismo de devaluación como metodología de ajuste, lo que no pueden hacer los países europeos porque tienen una moneda con tipo de cambio fijo.

* En la Región, el endeudamiento externo ayudó a postergar el agotamiento del modelo de sustitución de importaciones. En América el drama terminó. ¿Qué modelo caerá en Europa?, continente en el cual la tragedia recién comienza.

20. Toussaint, Eric ( 23 al 25 de mayo de 2003). La crisis de la deuda externa de América Latina en los siglos XIX y XX, documento presentado en el Seminario Internacional "América Latina y el Caribe: salir del impase de la deuda y del ajuste" organizado por el Comité para la Anulación de la Deuda del Tercer Mundo y por el Centro Nacional de la Cooperación al Desarrollo, llevado a cabo en Bruselas, 10 p.

* La dependencia de los países latinoamericanos respecto a los Estados Unidos se reflejó es que algunos países debieron comprar bonos del tesoro de ese país (bonos cupón cero) a fin de garantizar sus propios empréstitos en los mercados internacionales. Los países europeos ¿seguirán dependiendo de Goldman Sachs para garantizar sus obligaciones internacionales?

El escribir una completa historia de la deuda externa, al igual que hacerlo para la historia del teatro, abarcaría varios volúmenes y exigiría la amplia colaboración de expertos en una variedad de materias tales como la historia, la sicología, la antropología, la sociología, entre otras. La participación de jóvenes de todas las Universidades de América Latina, es necesaria, para junto con los expertos, escribir la historia permanente del pensamiento latinoamericano. 
Banco Interamericano de Desarrollo (Octubre de 1992). Progreso Económico y Social en América Latina Informe 1992. Washington D.C.

BBC Mundo-Economía. ¿Quien es responsable de la crisis en Grecia? (www.bbc.co.uk/mundo/economia

Becher, Joachim (2007). El endeudamiento externo de América Latina: un resumen histórico, en“El golpe del capital. Las crisis financieras en el Cono Sur y sus salidas". Montevideo: Editorial Coscoroba.

Fernández, Ángel (15 de septiembre de 2011). Instituto Juan de Mariana, www.juandemariana.or/comentario/5225/crisis/deuda/eurozona/

Hernández, Gladys. Evolución de la deuda externa de América Latina y el Caribe, en www.oid-ido.org, 1611-2005.

http://es.wikipedia.org/wiki/Crisis_de_la_deuda_soberana_europea.

Justo, Marcelo. BBC Mundo,www.bbc.co.uk/mundo/economía/2010/03/100325.

Maudos, Joaquín. (www.cincodias.com/articulo/ipinion/tragedia- griega-banca-europea).

Toussaint, Eric (23 al 25 de mayo de 2003). La crisis de la deuda externa de América Latina en los siglos XIX y XX, documento presentado en el Seminario Internacional "América Latina y el Caribe: salir del impase de la deuda y del ajuste" organizado por el Comité para la Anulación de la Deuda del Tercer Mundo y por el Centro Nacional de la Cooperación al Desarrollo, llevado a cabo en Bruselas, Bélgica.

www.espanol.rfi.rf/europa/20100502 “El eurogrupo da luz verde al plan de rescate”. 